Pacific Journal of Mathematics

THE NUMBER OF VECTORS JOINTLY ANNIHILATED BY
TWO REAL QUADRATIC FORMS DETERMINES THE INERTIA
OF MATRICES IN THE ASSOCIATED PENCIL

ANE UHLIG 


\title{
THE NUMBER OF VECTORS JOINTLY ANNIHILATED BY TWO REAL QUADRATIC FORMS DETERMINES THE INERTIA OF MATRICES IN THE ASSOCIATED PENCIL
}

\section{FrANK UHLIG}

\begin{abstract}
Pencils of real symmetric matrices and their associated quadratic forms are interrelated. It is well known that a pencil contains a definite matrix iff the associated quadratic forms do not vanish simultaneously, provided the matrices have dimension $n \geqq 3$. This knowledge is extended here to yield the following for nonsingular pairs of real symmetric matrices of dimension $n \geqq 3$ :

(I) The pencil $P(S, T)$ contains a semidefinite, but no definite matrix iff the maximal number $l$ of lin. ind. vectors simultaneously annihilated by the associated quadratic forms lies between 1 and $n-1$ and certain conditions on $S$ and $T$ hold if $l=n-1$.

(II) The pencil $P(S, T)$ contains only indefinite matrices iff $n-1 \leqq l \leqq n$ with other (complementary to the above) conditions holding if $l=n-1$.
\end{abstract}

First we introduce the relevant notation for a pair of real symmetric (r.s.) matrices $S$ and $T$ of the same dimension $n$ :

Definition 1. (a) The pencil $P(S, T)=\{a S+b T \mid a, b \in \boldsymbol{R}\}$ is a $d$-pencil if $P(S, T)$ contains a definite matrix.

(b) $P(S, T)$ is a s.d. pencil if $P(S, T)$ contains a nonzero semidefinite, but no definite matrix.

(c) $P(S, T)$ is an i-pencil if $P(S, T)$ contains only indefinite matrices, except for the zero matrix.

Notation. We denote by $Q_{S}$ the set $\left\{x \in \boldsymbol{R}^{n} \mid x^{\prime} S x=0\right\}$.

Definition 2. A pair of r.s. matrices $S$ and $T$ is called a nonsingular pair if $S$ is nonsingular.

This is our main result:

MAIN THEOREM. For a pair of r.s. matrices $S$ and $T$ of dimension $n \geqq 3$ let $l=\max \left\{k \mid\right.$ there exist $k$ lin. ind. vectors in $\left.Q_{S} \cap Q_{r}\right\}$. Then we have:

(a) $P(S, T)$ is a d-pencil iff $l=0$, and for a nonsingular pair $S, T$ :

(b) $P(S, T)$ is a s.d. pencil if and only if $1 \leqq l \leqq n-1$ and 
in case of $l=n-1$ we 'have that $S$ and $T$ are simultaneously congruent either to

(D): $\operatorname{diag}\left(\varepsilon\left(\begin{array}{ll}0 & 1 \\ 1 & 0\end{array}\right), \varepsilon_{3}, \cdots, \varepsilon_{n}\right)$ and $\operatorname{diag}\left(\eta\left(\begin{array}{ll}0 & \alpha \\ \alpha & 1\end{array}\right), \varepsilon_{3} \alpha, \cdots, \varepsilon_{n} \alpha\right)$ with $\alpha \in \boldsymbol{R}, \varepsilon, \eta, \varepsilon_{j}= \pm 1$ so that $\varepsilon_{m} \varepsilon_{k}=-1$ for at least one pair of indices $3 \leqq m, k \leqq n$, or to

(E): $\operatorname{diag}\left(\varepsilon_{1}, \cdots, \varepsilon_{n}\right)$ and $\operatorname{diag}\left(\varepsilon_{1} \lambda, \cdots, \varepsilon_{n-1} \lambda, \varepsilon_{n} \mu\right)$,

where $\varepsilon_{j}= \pm 1$ with $\varepsilon_{m} \varepsilon_{k}=-1$ for at least one pair $1 \leqq m, k \leqq n-1$, and $\lambda, \mu \in \boldsymbol{R}$ with $\lambda \neq \mu$.

(c) $P(S, T)$ is an $i$-pencil if and only if $n-1 \leqq l \leqq n$ and in case of $l=n-1$ we have that $S$ and $T$ are simultaneously congruent to

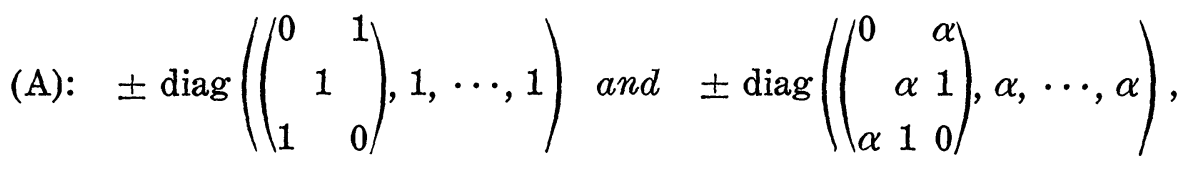

or

(B): $\quad \pm \operatorname{diag}\left(\left(\begin{array}{ll}0 & 1 \\ 1 & 0\end{array}\right), 1, \cdots, 1\right)$ and $\pm \operatorname{diag}\left(\left(\begin{array}{rr}b & a \\ a & -b\end{array}\right), \alpha, \cdots, a\right)$

with $a, b, \alpha \in R, b \neq 0$.

Part (a) is well known and was first proved to this extent by Calabi [1]; for a complete bibliography regarding (a) the reader should refer to the remarks in Uhlig [2], Theorem 0.2 .

Parts (b) and (c) will be proved with the help of the following theorems:

Theorem 1. (Uhlig [2]).

Let $S, T$ be a nonsingular pair of r.s. matrices of dimension greater than 2.

Then $P(S, T)$ is a s.d. pencil iff.

Either $S$ and $T$ are simultaneously congruent to diag $\left(a_{i}\right)$ and $\operatorname{diag}\left(b_{i}\right)$ with $b_{i} / a_{i} \neq b_{j} / a_{j}$ for at least one pair of indices $(i, j)$ and we have
(i) $\max _{a_{i}>0} \frac{b_{i}}{a_{i}}=\max _{a_{i}<0} \frac{b_{i}}{a_{i}}$ or
(ii) $\min _{a_{i}>0} \frac{b_{i}}{a_{i}}=\min _{a_{i}<0} \frac{b_{i}}{a_{i}}$,

or (iii) $S$ and $T$ are simultaneously congruent to $\operatorname{diag}\left(\varepsilon E, \cdots, \varepsilon E, \varepsilon_{k+1}, \cdots, \varepsilon_{j}, a_{j+1}, \cdots, a_{n}\right)$ and $\operatorname{diag}\left(\varepsilon E J, \cdots, \varepsilon E J, \varepsilon_{k+1} \alpha, \cdots, \varepsilon_{j} \alpha, b_{j+1}, \cdots, b_{n}\right)$, 
where $J=\left(\begin{array}{ll}\alpha & 1 \\ 0 & \alpha\end{array}\right), E=\left(\begin{array}{ll}0 & 1 \\ 1 & 0\end{array}\right) ; \varepsilon, \varepsilon_{i}= \pm 1, \alpha \in R, b_{l} \neq \alpha a_{l}$ and $\varepsilon, b_{l}-\alpha a_{l}$ all have the same sign for $l=j+1, \cdots, n$.

\section{THEOREM 2. (Uhlig [3]).}

Let $S, T$ be a nonsingular pair of r.s. matrices of dimension greater than 2. Let $J$ be the real Jordan normal form of $S^{-1} T$ and define $l=\max \left\{k \mid\right.$ there exist $k$ lin. ind. vectors in $\left.Q_{S} \cap Q_{T}\right\}$.

If $(\alpha)$ : $J$ contains one 3-dimensional Jordan block, linear blocks else for the same eigenvalue and inertia $S=(n-1,1,0)$ or $(1, n-1,0)$.

Or ( $\beta$ ): $J$ contains one 2-dimensional Jordan block corresponding to a nonreal eigenvalue, linear blocks else for identical eigenvalues and inertia $S=(n-1,1,0)$ or $(1, n-1,0)$, then $l=n-1$.

If $(\gamma): J$ contains $k \geqq 1$ identical 2-dimensional Jordan blocks corresponding to one real eigenvalue $\lambda$, linear blocks else for eigenvalues $\mu_{i}(i=2 k+1, \cdots, n)$ and the set

$$
\left\{\varepsilon_{1}, \cdots, \varepsilon_{k}, \varepsilon_{i}\left(\mu_{i}-\lambda\right) \mid i>2 k\right\} \text {, }
$$

where the $\varepsilon_{i}= \pm 1$ are the constants in the canonical pair form of $S$ and $T$, contains $r \geqq 0$ zeroes $\mu_{2 k+1}-\lambda=\cdots=\mu_{2 k+r}-\lambda=0$ and only positive or only negative numbers else, and $\varepsilon_{2 k+1}=\cdots=\varepsilon_{2 k+r}$, then $l=k$.

If $(\delta)$ : Condition $(\gamma)$ holds except that not all $\varepsilon_{i}$ are the same for $2 k+1 \leqq i \leqq 2 k+r$, then $l=k+r$.

If $(\eta): S$ and $T$ can be simultaneouly diagonalised by a real congruence transformation, then $l=0,2,3, \cdots, n$ are possible, depending on $S$ and $T$.

In all other cases $Q_{S} \cap Q_{T}$ contains $l=n$ lin. ind. vector.

And

\section{THEOREM 3. (Uhlig [3]).}

Let $S, T, J, n, l, k, r,(\alpha),(\beta),(\gamma),(\delta),(\eta)$ be as in Theorem 2.

If $l=0$, then $(\eta)$ holds for $J$.

If $l=1$, then $(\gamma)$ holds for $J$ with $k=1$.

If $2 \leqq l \leqq n-2$, then $(\gamma),(\delta)$ or $(\eta)$ holds for $J$. for $J$.

If $l=n-1$, then $(\alpha),(\beta),(\delta)($ with $k=1, r=n-2)$, or $(\eta)$ holds

If $l=n$, then none of $(\alpha),(\beta),(\gamma)$ or $(\delta)$ may hold for $J$.

Proof of the Main Theorem.

Part (b): All s.d. pencils have been characterized in Theorem 1. So if $S$ and $T$ are simultaneously congruent to $D_{1}=\operatorname{diag}\left(\varepsilon_{i}\right)$ and $D_{2}=$ diag $\left(\varepsilon_{i} \lambda_{i}\right)$ then (i) or (ii) of Theorem 1 must hold. Assume (i) holds and let 


$$
\lambda=\max _{\varepsilon_{i}>0} \lambda_{i}=\max _{\varepsilon_{i}<0}\left(-\lambda_{i}\right) \text {. }
$$

Then $x^{\prime} D_{1} x=x_{m}^{2}-x_{k}^{2}+\sum \varepsilon_{i} x_{i}^{2}=0$ and $x^{\prime} D_{2} x=\lambda x_{m}^{2}-\lambda x_{k}^{2}+\sum \varepsilon_{i} \lambda_{i} x_{i}^{2}=$ 0 implies

$$
\sum_{i \neq m, k} \varepsilon_{i}\left(\lambda_{i}-\lambda\right) x_{i}^{2}=0
$$

Now for $\varepsilon_{i}>0$ we have $\lambda_{i} \leqq \lambda$ while $\varepsilon_{i}<0$.implies $\lambda_{i} \geqq \lambda$. Hence (2) is a semidefinite quadratic form in $x_{1}, \cdots, x_{n}$ with at least one nonzero coefficient $\varepsilon_{i}\left(\lambda_{i}-\lambda\right)$ by Theorem 1 . Thus all vectors $x \in Q_{D_{1}} \cap$ $Q_{D_{2}}$ must have their $i$ th component equal to zero for all $i$ with $\lambda_{i} \neq \lambda$ and hence $2 \leqq l \leqq n-1$ and $l=n-1$ exactly in case of $(\eta)$. The proof in case of (ii) is similar.

If $S$ and $T$ are not simultaneously diagonalizable, then by Theorem 1 we must have case $(\gamma)$ or $(\delta)$ of Theorem 2 . We conclude that $1 \leqq$ $l \leqq n-1$ and $l=n-1$ only if $(\delta)$ holds with $k=1, r=n-2$, hence if (D) holds. Now for the converse: If $l=n-1$ and (D) holds, then $P(S, T)$ is a s.d. pencil by Theorem 1 . If $1 \leqq l \leqq n-2$ then by Theorem 3 only $(\gamma),(\delta)$ or $(\eta)$ may hold for $S$ and $T$. We are done in case of $(\gamma)$ or $(\delta)$ due to Theorem 1. For arbitrary $l$ the case of $(\eta)$ will be settled by the following Lemma:

Lemma 1. For two real diagonal matrices $D_{1}=\operatorname{diag}\left(\varepsilon_{i}\right)$ and $D_{2}=$ $\operatorname{diag}\left(\varepsilon_{i} \lambda_{i}\right)$, where $\varepsilon_{i}= \pm 1, i=1, \cdots, n$ let $l=\max \{k\}$ there exist $k$ lin. ind. vectors in $\left.Q_{D_{1}} \cap Q_{D_{2}}\right\}$.

Then $P\left(D_{1}, D_{2}\right)$ is an $i$-pencil iff $l=n$.

Proof. Assume $P\left(D_{1}, D_{2}\right)$ is not an $i$-pencil. Then it must be a $d$-pencil or a $s . d$. pencil, in which cases $l=0$ or $1 \leqq l \leqq n-1$ as shown above.

Conversely if $P\left(D_{1}, D_{2}\right)$ is an $i$-pencil, then $L=\left\{\left(\varepsilon_{i}, \varepsilon_{i} \lambda_{i}\right)\right\}$ is contained in no open or closed halfspace of $R^{2}$ and hence we may assume without loss of generality that $\lambda_{3}<\lambda_{1}<\lambda_{2}$ and $\varepsilon_{1}=-1, \varepsilon_{2}, \varepsilon_{3}=1$.

Then the quadratic forms read like:

$$
x^{\prime} D_{1} x=-x_{1}^{2}+x_{2}^{2}+x_{3}^{2}+\sum_{i=3}^{n} \varepsilon_{i} x_{i}^{2}
$$

and

$$
x^{\prime} D_{2} x=-\lambda_{1} x_{1}^{2}+\lambda_{2} x_{2}^{2}+\lambda_{3} x_{3}^{2}+\sum_{i=3}^{n} \varepsilon_{i} \lambda_{i} x_{i}^{2} .
$$

Let $f: \boldsymbol{R}^{3} \rightarrow \boldsymbol{R}^{2} ; f\left(x_{1}, x_{2}, x_{3}\right)=\left(-x_{1}^{2}+x_{2}^{2}+x_{3}^{2},-\lambda_{1} x_{1}^{2}+\lambda_{2} x_{2}^{2}+\lambda_{3} x_{3}^{2}\right)$. We will show that $f$ is surjective: 
First if $a, b \in \operatorname{Im} f$, then $\alpha a+\beta b \in \operatorname{Im} f$ for all $\alpha, \beta \geqq 0$.

For if $f\left(x_{1}, x_{2}, x_{3}\right)=a, f\left(y_{1}, y_{2}, y_{3}\right)=b$, then

$$
f\left(\sqrt{\alpha x_{1}^{2}+\beta y_{1}^{2}}, \sqrt{\alpha x_{2}^{2}+\beta y_{2}^{2}}, \sqrt{\left.\alpha x_{3}^{2}+\beta y_{3}^{2}\right)}=\alpha a+\beta b .\right.
$$

Secondly $0 \neq x \in \boldsymbol{R}^{3}$ can always be found s.t. $f(x)=(\varepsilon, \delta)$, whenever $(\varepsilon, \delta) \in\{(0,0),(1,0),(-1,0),(0,1),(0,-1)\}$, which will complete the proof that $\operatorname{Im} f=\boldsymbol{R}^{2}$ :

To solve $f(x)=(\varepsilon, \delta)$ means to solve the following linear system of equations in $x_{1}^{2}, x_{2}^{2}, x_{3}^{2}$ :

$$
\left(\begin{array}{lll|l}
-1 & 1 & 1 & \varepsilon \\
-\lambda_{1} & \lambda_{2} & \lambda_{3} & \delta
\end{array}\right)
$$

This is equivalent to

$$
\left(\begin{array}{ccc|c}
-1 & 1 & 1 & \varepsilon \\
0 & \lambda_{2}-\lambda_{1} & \lambda_{3}-\lambda_{1} & \delta-\varepsilon \lambda_{1}
\end{array}\right)
$$

Since $\lambda_{3}<\lambda_{1}<\lambda_{2}$, the second equation in (3) represents a line $L$ with positive slope in the quarter plane $\left\{\left(x_{2}^{2}, x_{3}^{2}\right)\right\}$. Hence this line will have points in common with the set $A=\left\{\left(x_{2}^{2}, x_{3}^{2}\right) \mid x_{2}^{2}+x_{3}^{2}>1\right\}$. But $\varepsilon \leqq 1$, hence taking $\left(x_{2}^{2}, x_{3}^{2}\right) \in L \cap A \neq \varnothing$, then the system (3) can be solved with $x_{1}^{2}>0$.

The lemma is proved if we exhibit $n$ lin. ind. vectors in $Q_{D_{1}} \cap Q_{D_{2}}$. Let $y_{1}=\left(x_{1}, x_{2}, x_{3}, 0, \cdots, 0\right)$ with $x_{1}, x_{2}, x_{3} \neq 0$ s.t. $f\left(x_{1}, x_{2}, x_{3}\right)=0$,

$$
\begin{aligned}
& y_{2}=\left(-x_{1}, x_{2}, x_{3}, 0, \cdots, 0\right) \\
& y_{3}=\left(-x_{1}-x_{2}, x_{3}, 0, \cdots, 0\right) \text { and } \\
& y_{i}=\left(\alpha_{i}, \beta_{i}, \gamma_{i}, 0, \cdots, 0\right)+e_{i} \text { for } i \geqq 4,
\end{aligned}
$$

where $e_{i}$ is the $i$ th unit vector and $\alpha_{i}, \beta_{i}, \gamma_{i}$ are such that $f\left(\alpha_{i}, \beta_{i}, \gamma_{i}\right)=$ $\left(-\varepsilon_{i},-\varepsilon_{i} \lambda_{i}\right)$.

Part (c): This is obvious once parts (a) and (b) have been proved. Thus the Main Theorem is proved.

Outlook. The Main Theorem contains three "if and only if" statements relating the type of pencil a nonsingular pair of r.s. matrices $S$ and $T$ generates to the maximal number $l$ of lin.ind. vectors simultaneously annihilated by the two associated real quadratic forms. On the one hand the distinction of $d$-, s.d. and $i$-pencils is easily made, while on the other it is also enough to know $l$ for a specific pair of matrices except for the case that $l=n-1$. Then the further conditions on the finest simultaneous block diagonal structure of $S$ and $T$ are somewhat hard to verify; one needs to know the real Jordan normal form of $S^{-1} T$ for example. 
It is an open question whether this difficulty could be avoided either by changing the concepts of $d$-, s.d. or $i$-pencil slightly or by using a characterization of pairs of quadratic forms different from our number $l$.

Acknowledgment. Dr. Olga Taussky-Todd, my thesis advisor at the California Institute of Technology, had suggested to try to generalize the theorems about simultaneous diagonalization and about $d$-pencils by studying $Q_{S} \cap Q_{T}$. Moreover, Dr. H. F. Bohnenblust suggested to find properties of $P(S, T)$ that relate to $l$, the maximal number of lin. ind. vectors in $Q_{S} \cap Q_{T}$, rather than relating $l$ to the real Jordan normal form of $S^{-1} T$ as done in [3]. I am grateful to both, for both suggestions have been intertwined here.

\section{REFERENCES}

1. E. Calabi, Linear systems of real quadratic forms, Proc. Amer. Math. Soc., 15 (1964), 844-846.

2. F. Uhlig, Definite and semidefinite matrices in a real symmetric matrix pencil, Pacific J. Math., 49 (1973), 561-568.

3. - On the maximal number of linearly independent real vectors simultaneously annihilated by two real quadratic forms, Pacific J. Math., 49 (1973), 543-560.

Received July 12, 1972.

UNIVERSITÄT WÜRZBURG 


\section{PACIFIC JOURNAL OF MATHEMATICS}

\section{EDITORS}

RICHARD ARENS (Managing Editor)

University of California

Los Angeles, California 90024

\section{R. A. Beaumont}

University of Washington

Seattle, Washington 98105

\section{J. DuGundJI*}

Department of Mathematics

University of Southern California

Los Angeles, California 90007

D. Gilbarg and J. Milgram

Stanford University

Stanford, California 94305

ASSOCIATE EDITORS
E. F. BECKENBACH
B. H. NeumanN
F. WOLF
K. YoSHIDA

\section{SUPPORTING INSTITUTIONS}

UNIVERSITY OF BRITISH COLUMBIA

CALIFORNIA INSTITUTE OF TECHNOLOGY

UNIVERSITY OF CALIFORNIA

MONTANA STATE UNIVERSITY

UNIVERSITY OF NEVADA

NEW MEXICO STATE UNIVERSITY

OREGON STATE UNIVERSITY

UNIVERSITY OF OREGON

OSAKA UNIVERSITY

\author{
UNIVERSITY OF SOUTHERN CALIFORNIA \\ STANFORD UNIVERSITY \\ UNIVERSITY OF TOKYO \\ UNIVERSITY OF UTAH \\ WASHINGTON STATE UNIVERSITY \\ UNIVERSITY OF WASHINGTON \\ AMERICAN MATHEMATICAL SOCIETY \\ NAVAL WEAPONS CENTER
}

The Supporting Institutions listed above contribute to the cost of publication of this Journal, but they are not owners or publishers and have no responsibility for its content or policies.

Mathematical papers intended for publication in the Pacific Journal of Mathematics should be in typed form or offset-reproduced, (not dittoed), double spaced with large margins. Underline Greek letters in red, German in green, and script in blue. The first paragraph or two must be capable of being used separately as a synopsis of the entire paper. Items of the bibliography should not be cited there unless absolutely necessary, in which case they must be identified by author and Journal, rather than by item number. Manuscripts, in duplicate if possible, may be sent to any one of the four editors. Please classify according to the scheme of Math. Rev. Index to Vol. 39. All other communications to the editors should be addressed to the managing editor, or Elaine Barth, University of California, Los Angeles, California, 90024.

50 reprints are provided free for each article; additional copies may be obtained at cost in multiples of 50 .

The Pacific Journal of Mathematics is issued monthly as of January 1966. Regular subscription rate: $\$ 48.00$ a year (6 Vols., 12 issues). Special rate: $\$ 24.00$ a year to individual members of supporting institutions.

Subscriptions, orders for back numbers, and changes of address should be sent to Pacific Journal of Mathematics, 103 Highland Boulevard, Berkeley, California, 94708.

\section{PUBLISHED BY PACIFIC JOURNAL OF MATHEMATICS, A NON-PROFIT CORPORATION}

Printed at Kokusai Bunken Insatsusha (International Academic Printing Co., Ltd.), 270, 3-chome Totsuka-cho, Shinjuku-ku, Tokyo 160, Japan.

* C. R. DePrima California Institute of Technology, Pasadena, CA 91109, will replace J. Dugundji until August 1974.

Copyright (C) 1973 by

Pacific Journal of Mathematics

All Rights Reserved 


\section{Pacific Journal of Mathematics}

\section{Vol. 49, No. $2 \quad$ June, 1973}

Wm. R. Allaway, On finding the distribution function for an orthogonal polynomial

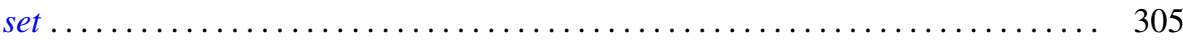

Eric Amar, Sur un théorème de Mooney relatif aux fonctions analytiques bornées... . 311

Robert Morgan Brooks, Analytic structure in the spectrum of a natural system . . . . 315

Bahattin Cengiz, On extremely regular function spaces . . . . . . . . . . . . . . 335

Kwang-nan Chow and Moses Glasner, Atoms on the Royden boundary . . . . . . . . . 339

Paul Frazier Duvall, Jr. and Jim Maxwell, Tame $Z^{2}$-actions on $E^{n} \ldots \ldots \ldots \ldots \ldots . .349$

Allen Roy Freedman, On the additivity theorem for $n$-dimensional asymptotic

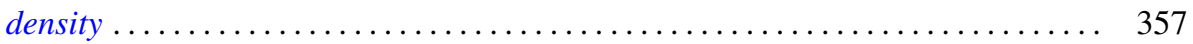

John Griffin and Kelly Denis McKennon, Multipliers and the group $L_{p}$-algebras . . . 365

Charles Lemuel Hagopian, Characterizations of $\lambda$ connected plane continua ....... 371

Jon Craig Helton, Bounds for products of interval functions . . . . . . . . . . . 377

Ikuko Kayashima, On relations between Nörlund and Riesz means . . . . . . . . . . 391

Everett Lee Lady, Slender rings and modules . . . . . . . . . . . . . . . . . . 397

Shozo Matsuura, On the Lu Qi-Keng conjecture and the Bergman representative

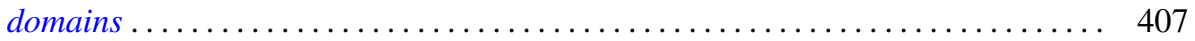

Stephen H. McCleary, The lattice-ordered group of automorphisms of an $\alpha$-set . . . 417

Stephen H. McCleary, o-2-transitive ordered permutation groups .......... 425

Stephen H. McCleary, o-primitive ordered permutation groups. II . . . . . . . . . 431

Richard Rochberg, Almost isometries of Banach spaces and moduli of planar

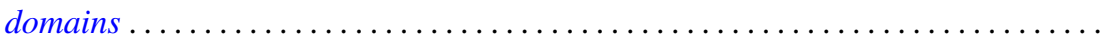

R. F. Rossa, Radical properties involving one-sided ideals . . . . . . . . . . . . . 467

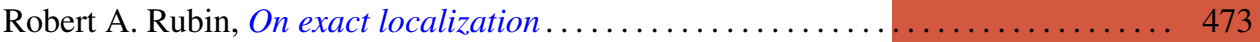

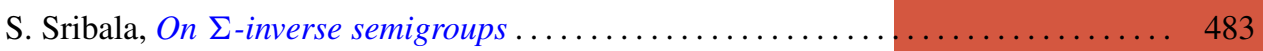

H. M. (Hari Mohan) Srivastava, On the Konhauser sets of biorthogonal polynomials suggested by the Laguerre polynomials ...................... 489

Stuart A. Steinberg, Rings of quotients of rings without nilpotent elements . ...... 493

Daniel Mullane Sunday, The self-equivalences of an $H$-space . . ............ 507

W. J. Thron and Richard Hawks Warren, On the lattice of proximities of $\check{C} e c h$

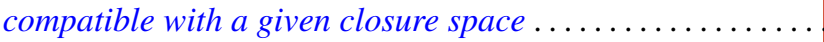

Frank Uhlig, The number of vectors jointly annihilated by two real quadratic forms determines the inertia of matrices in the associated pencil .

Frank Uhlig, On the maximal number of linearly independent real vectors annihilated simultaneously by two real quadratic forms ..............

Frank Uhlig, Definite and semidefinite matrices in a real symmetric matrix pencil . . 561

Arnold Lewis Villone, Self-adjoint extensions of symmetric differential operators . . . 569

Cary Webb, Tensor and direct products . ....................... 579

James Victor Whittaker, On normal subgroups of differentiable

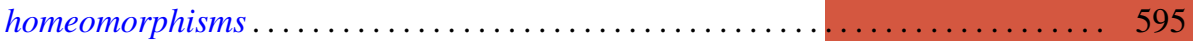

Jerome L. Paul, Addendum to: "Sequences of homeomorphisms which converge to

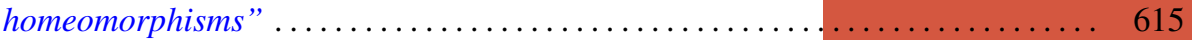

David E. Fields, Correction to: “Dimension theory in power series rings” ........ 616

Peter Michael Curran, Correction to: “Cohomology of finitely presented groups”. . . 617

Billy E. Rhoades, Correction to: “Commutants of some Hausdorff matrices” ...... 617

Charles W. Trigg, Corrections to: "Versum sequences in the binary system” ...... 619 